\title{
RADIO NOISE MEASUREMENTS ON THE PASSAGE OF SOLAR PARTICLES THROUGH THE CORONA
}

\author{
T. R. HARTZ
}

\section{Radio Physics Laboratory, Defence Research Telecommunications Establishment Defence Research Board, Ottawa, Canada}

The swept-frequency receiver is one of the most powerful tools for studying the processes that go on in the sun's atmosphere. However, the instrumentation becomes fairly elaborate if a large frequency range is to be covered by such an apparatus. Consequently, only a few such receivers have been built, and those that are in operation are somewhat limited in their frequency coverage. It then seems worth while to examine the data from existing fixedfrequency receiving stations in order to determine the extent to which they can supplement or approximate the results of the swept-frequency apparatus. Many solar observations at fixed frequencies have been made and are still being made by numerous observers. These data contain much meaningful information if they can be interpreted successfully.

At the Radio Physics Laboratory in Ottawa solar-noise recordings are being made at 500,50 , and $30 \mathrm{Mc} / \mathrm{s}$. These observations are being supplemented

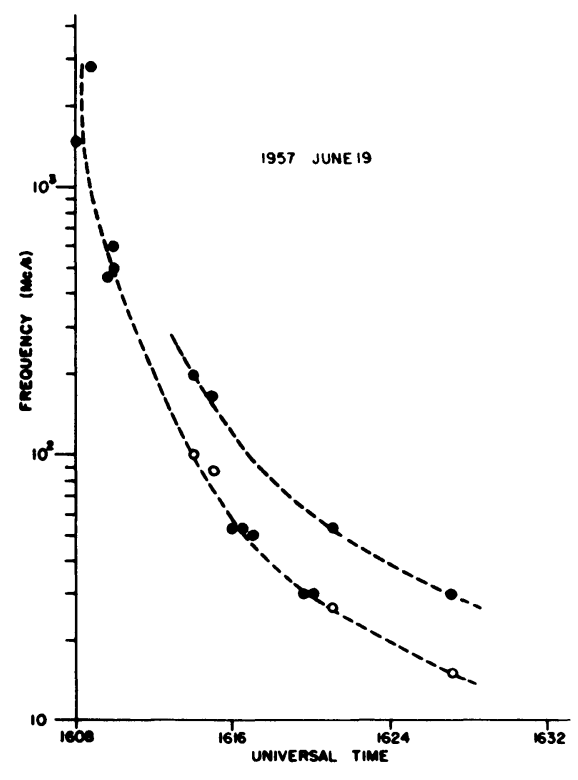

Fig. 1. Slow-drift noise bursts associated with a solar flare of 1957 June 19. with readily available data from other observatories, from the International Geophysical Year data centers, and from the I.A.U. Quarterly Bulletin of Solar Activity. This technique has rather limited application since the details on the respective records are not available. Nevertheless, such information as the starting time of the noise bursts at the different frequencies is very useful. An accuracy of not better than plus or minus one minute can be assumed, but in many cases this is adequate for meaningful interpretation.

The noise bursts accompanying solar flares can be interpreted in terms of an initiating disturbance moving outward through the corona. Fig. 1 shows a graph of the starting time of the bursts that accompanied an importance $2+$ flare on 1957 June 19. The double line was suggested by 
two separate bursts that were clearly discernible on our records at the lower frequencies. On the assumption that the two bursts are harmonically related, this graph can be taken as a type II burst on the usual classification. In the diagram the full circles show the actual observations, and the open circles represent the same observation at the half-frequency.

The procedure is admittedly hazardous since errors in interpretation can easily arise. It has been used on our own recordings of 50 and $30 \mathrm{Mc} / \mathrm{s}$ almost exclusively because the full record was available to assist in the interpretation. It permits the inference of solar data at frequencies as low as $15 \mathrm{Mc} / \mathrm{s}$ where such direct observations are often impossible because of the ionospheric cut-off.

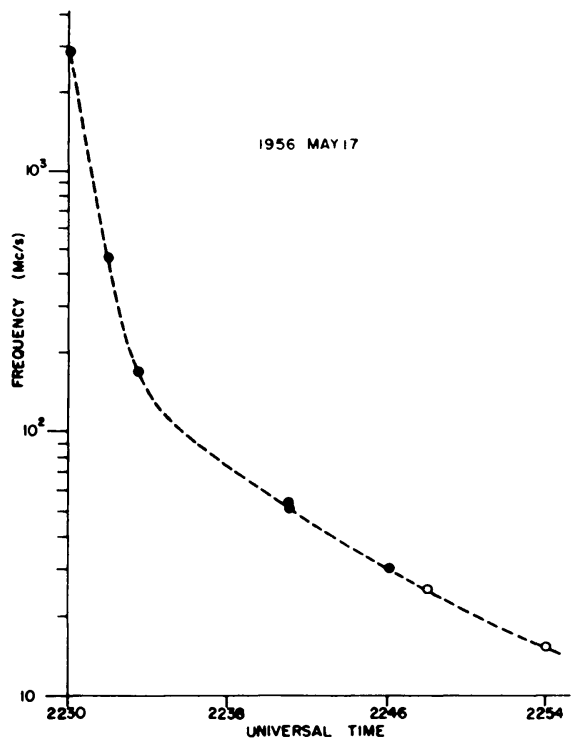

FIg. 2. Slow-drift noise bursts associated with a solar flare of 1956 May 17.

Fig. 2 shows another example of such a slow drift-curve. As before, the starting times of the bursts at the different frequencies are given, with the open circles being inferred as sub-harmonic observations. This case is for an importance 3 flare of 1956 May 17 . The information has been turned into a measurement of the disturbing mechanism's outward velocity as a function of height in the corona; this is shown in Fig. 3. To derive this curve the noise burst is assumed to start when the generating mechanism reaches the envelope from within which no radiation at that particular frequency can reach the earth; i.e., the so-called escape level. This is the envelope of the ray trajectories as computed by Jaeger and Westfold [1] and is illustrated in Fig. 4.

Fig. 3 shows that lower velocities are obtained at lower rather than at greater heights. It is recognized that the shape of this curve depends on the accuracy of timing at the higher frequencies, but a straight vertical line is impossible to achieve with the reported observations unless the identification of the bursts is in error. One is then led to conclude that an acceleration of the disturbance takes place in the corona, and that the magnitude of the deduced velocity depends on the frequency range under consideration. In an early paper, Wild [2] presented evidence for acceleration of the disturbing agency, though his observations covered a smaller frequency range than ours cover.

It is interesting to note that the curve tends toward a constant velocity at heights of about one solar radius. Our observations at 30 and $50 \mathrm{Mc} / \mathrm{s}$ have been used to establish this terminal velocity in as many of these slow-drift cases as possible. On the assumption that the traveling disturbance is a cloud of particles, we have also examined the conditions in the earth's iono- 


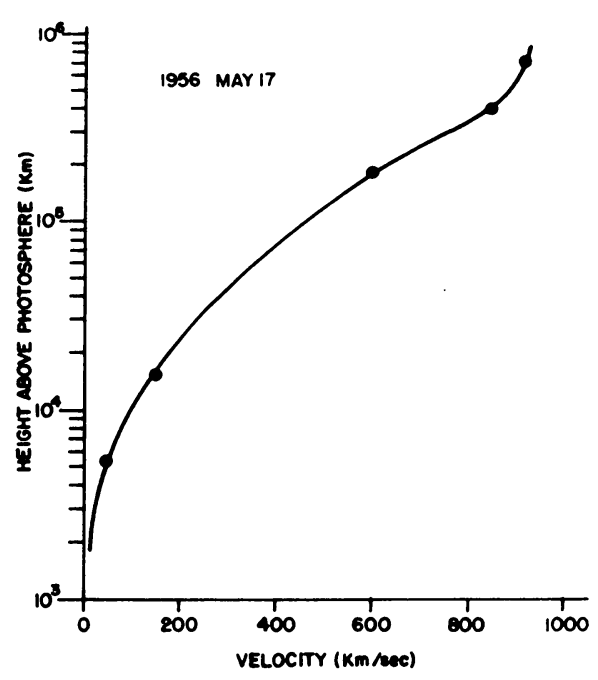

FIG. 3. Velocity of the outward-traveling disturbances as a function of height in the solar corona.

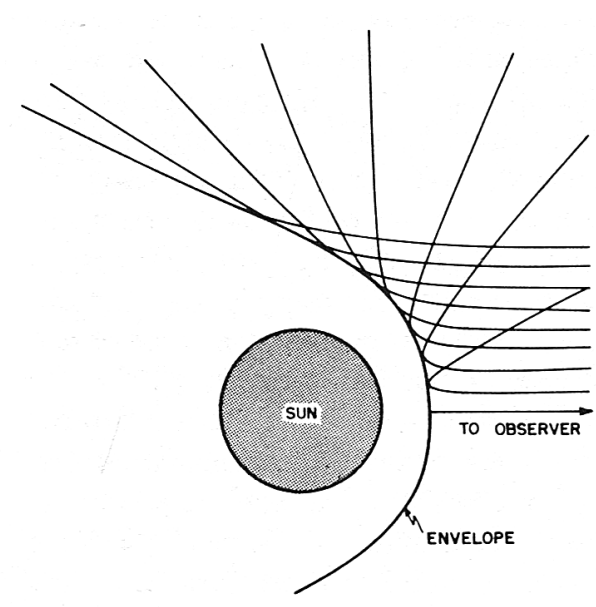

Fig. 4. Ray trajectories at $52 \mathrm{Mc} / \mathrm{s}$ based on values from Jaeger and Westfold [1]. The location of the envelope from within which radiation cannot reach the earth is shown.

sphere following the solar flares. In many cases magnetic storms, great auroral displays, and other ionospheric effects have been observed to follow the flare with a delay almost exactly what we expected from the deduced terminal velocity. There now seems no doubt that those flares that are followed by the distinctive slow-drift noise bursts of large magnitude are the origin of the particles that produce the terrestrial effects some two days later. Consequently, the bursts of radio noise are produced in the corona by the traveling particles in the vicinity of the escape envelope for that particular frequency.

These results are for those noise bursts that are very large and distinctive, and that are observed over a wide range of frequencies $(3000$ to $30 \mathrm{Mc} / \mathrm{s})$. In over three-fourths of the two dozen cases that have now been observed, the time delay between the flare and the geomagnetic storm as predicted from the deduced particle velocities agrees to within a few hours with the actual observed delay.

It is also possible to use fixed-frequency observations to distinguish rapiddrift bursts that might possibly be identified elsewhere as type III bursts. Fig. 5 shows such an example in the case of an importance 2+ flare of 1957 September 12. Large-amplitude noise bursts are observed at all frequencies in the range from 3000 to $30 \mathrm{Mc} / \mathrm{s}$. It is immediately obvious that timing inaccuracies set a real limit in these cases, since the bursts appear almost simultaneously at all frequencies. Measurements at 50 and $30 \mathrm{Mc} / \mathrm{s}$, where one might expect the longest delays, show that there is usually some slope to the curve, but in no case has a velocity less than about $5000 \mathrm{~km} / \mathrm{second}$ been obtained. This limit is set by the experimental errors in timing the bursts. 


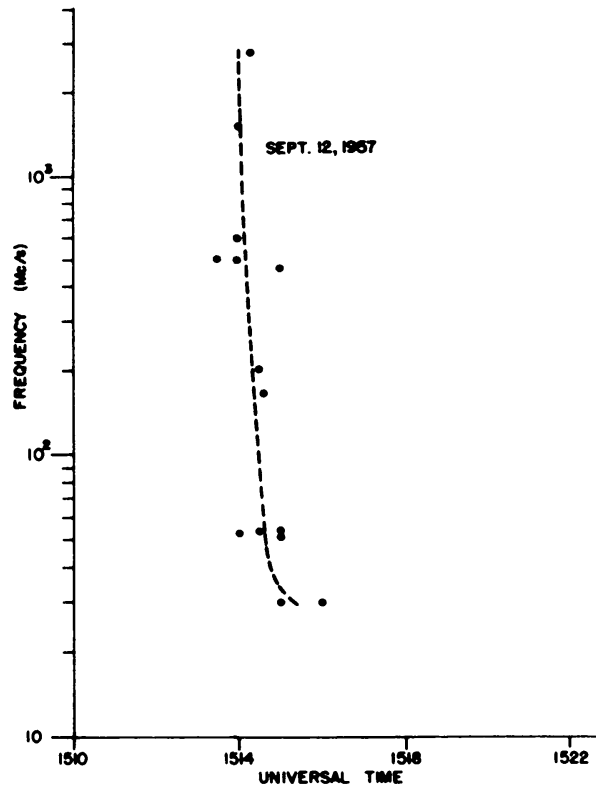

FIG. 5. Fast-drift noise bursts associated with a solar flare of 1957 September 12 .
It has been suggested that if particles are responsible for these rapid bursts, the great velocities would be consistent with cosmic rays. However, in the past twenty-one years only five cases have occurred in which a cosmicray increase was observed to follow a flare. The explanation would seem to lie elsewhere, presumably with particles of lesser energy which would not penetrate to the earth's surface.

Bailey [3] first reported an ionospheric effect due to such high-velocity particles for the great flare of 1956 February 23. Since then we have observed similar effects in connection with other flares for which such rapiddrift bursts have been observed. The effect is a characteristic absorption of radio waves in the vicinity of the auroral zone, which commences a few hours after the flare. In such cases there is no recognizable magnetic or auroral effect. In each case the time delay is consistent with particle velocities of the order of $10^{4} \mathrm{~km} / \mathrm{second}$. The penetration into the ionosphere, perhaps as deep as the $70 \mathrm{~km}$ level, is also consistent with ions traveling at such velocities. So far the particles have not been identified but arguments exist favoring either protons or calcium ions.

For the particular example given in Fig. 5, the unusual high-latitude absorption started about 6 hours after the flare, corresponding to a particle velocity of about $7000 \mathrm{~km} / \mathrm{second}$, which is not inconsistent with the shape of the curve sketched in the diagram.

It is concluded that both the slow-drift bursts and the rapid-drift bursts can be linked directly with subsequent terrestrial effects that are produced by particle influx. Consequently, the traveling particle cloud must be taken as the noise-producing agency in each case.

Likewise, the close agreement in specific cases between the sun-to-earth travel time of the particles, based on the velocity deduced from the slow-drift bursts, and the observed delay between the flare and the magnetic storm means that the region of origin has been correctly identified. The noise burst originates at the envelope of the ray trajectories, directly over the flare.

Recently, we obtained some measurements that provide direct evidence of this. By operating a large phase-sweeping interferometer at $52 \mathrm{Mc} / \mathrm{s}$ somewhat similar to the equipment used by Payne-Scott and Little at $97 \mathrm{Mc} / \mathrm{s}$ [4], we have been able to observe the angle of arrival of the noise bursts at this frequency with an accuracy of about \pm 2 minutes of arc. This is sufficient 
to establish the region of origin in those cases when the flare occurs at an appreciable distance from the center of the solar disk. So far our results for several small flares place the burst origin on or very close to the envelope. However, the results are still preliminary since the apparatus has only been in operation for about 3 months, and not enough flares have occurred at the right time and in the proper position on the sun.

The location of the burst source at the escape envelope suggests that the peculiar properties of this envelope must be carefully considered in connection with the generation mechanism. At least two important properties are immediately evident and have been discussed before: the envelope is the most favorable position available for observing a band of noise centered on the local plasma frequency, and it provides focusing in the direction of observation.

The angle of arrival measurements for a noise storm, as opposed to a burst, provide an entirely different answer. Our measurements have shown that noise storms, which are apparently associated with sunspots, originate over the spot group but at a height considerably greater than would correspond to the envelope. While our results are still inconclusive, we have tried to obtain observations for noise storms associated with spot groups near the solar limb. For example, one small storm gave a height of about $10^{\circ} \mathrm{km}$ when the associated spot was about 42 degrees off the center of the apparent disk. The envelope height for these conditions would be only about $3 \times 10^{5}$ above the photosphere. We are continuing our observations in this regard.

Another study in connection with solar flares has shown promising results; e.g., the large noise increase that is observed to follow the burst in the case of some flares. An example of this is shown in Fig. 6, for the flare of 1957 June 19. The diagram shows the record obtained at $30 \mathrm{Mc} / \mathrm{s}$ with a fairly broad-beam antenna. Under these conditions the chart shows a combination of cosmic and solar noise. The SID, or sudden cosmic-noise fade, can be seen in the recording at the time of the flare, and very soon after, one can see the start of the slow-drift burst at this particular frequency. The second burst has been regarded as caused by harmonic generation at half the frequency. However, attention is directed to the noise increase that follows the

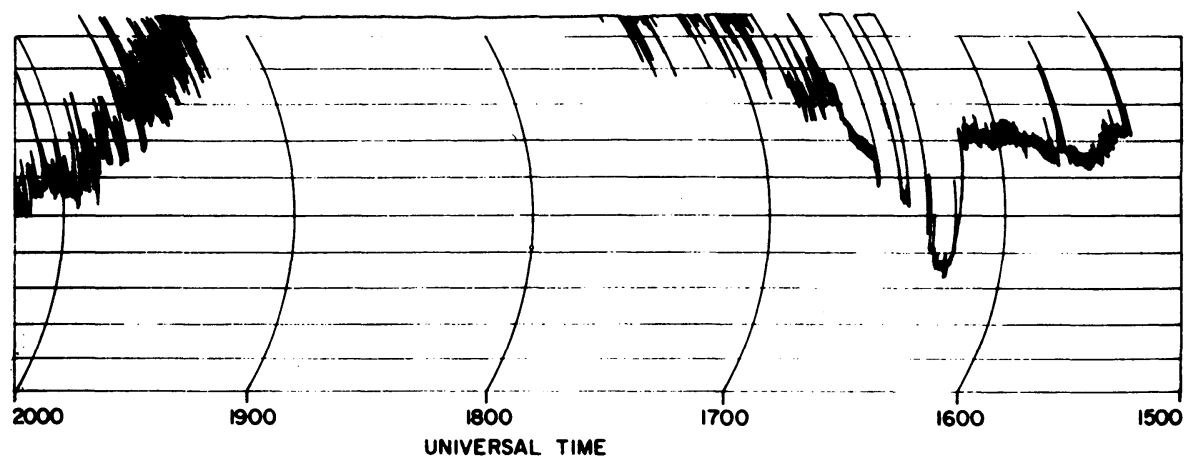

FIG. $6.30 \mathrm{Mc} / \mathrm{s}$ noise recording following the flare that started at $16^{\mathrm{h}} 08^{\mathrm{m}}$ U.T. on 1957 June 19. 
bursts and persists for several hours. This feature when it appears at higher frequencies has been called the type IV burst, but I shall consider it as a noise increase or noise storm associated with a flare.

We noted that this type of noise storm seems to occur only when the deduced velocity for the particle cloud is of the order of $1000 \mathrm{~km} / \mathrm{second}$ or less. When the particle velocities are higher this feature seems to be missing, which suggests that this particular phase of the flare-associated event is produced in some way by the slower particles, possibly by the fall back into the sun of the low-velocity part of the corpuscular cloud.

The method of determining particle velocities by using the starting time of the bursts probably yields the velocity of the fastest particles in the cloud, which might be expected to be in the leading edge. If a distribution of velocities exists behind this leading edge, it is not inconceivable that some particles should have speeds low enough to prohibit their escape from the sun's gravity. In consequence they will fall back into the corona, where their presence may be noted through the noise storm they produce. One would then expect the effect to be noticed only for particle-cloud velocities slightly greater than the escape velocity, as is indeed the case.

\section{CONCLUSIONS}

1. It is possible to interpret results from fixed-frequency receivers to provide information on solar particles traveling through the corona. This technique can be used over a wide range of frequencies with certain limitations to approximate the results of a swept-frequency receiver.

2. By association with observed terrestrial effects, it has been shown that both the slow-drift bursts and the fast-drift bursts are produced by particles traveling in the corona following a solar flare.

3. The agreement between the velocity deduced from an observed sun-toearth delay time and the velocity deduced from the noise bursts is sufficiently close to establish the source of the noise bursts at the envelope of the ray trajectories in the corona.

4. The preliminary results of a high-resolution $52 \cdot \mathrm{Mc} / \mathrm{s}$ phase-sweeping interferometer also support the conclusion that the noise bursts originate at the envelope.

5. Sunspot-associated noise storms at $52 \mathrm{Mc} / \mathrm{s}$ seem to occur at considerably greater heights in the corona.

6. The noise-increase phase of the flare-associated radio event can be explained if a distribution of particle velocities exists in the corpuscular cloud. It is suggested that the noise increase results from the fall back into the corona of some of the slower particles.

\section{REFERENCES}

[1] Jaeger, J. C., and Westfold, K. C. Aust. J. Sci. Res. A 3, 376, 1950.

[2] Wild, J. P. Aust. J. Sci. Res. A 3, 399, 1950.

[3] Bailey, D. K. J. Geophys. Res. 62, 431, 1957.

[4] Payne-Scott, Ruby, and Little, A. G. Aust. J. Sci. Res. A 4, 508, 1951. 


\section{Discussion}

Takakura: In our dynamic spectrometer, type III and type II bursts generally did not extend above $600 \mathrm{Mc} / \mathrm{s}$. The outbursts at the centimeter region seem to be distinct from type II, and are probably type IV. Therefore, it is dangerous to extend the drift-curve of frequencies up to the centimeter region.

Hartz: I realize that the identification of corresponding features over a wide range of frequencies is often very difficult. The character of the radiation may change appreciably from one end of the spectrum to the other. We have confined our considerations to the very large outbursts that can be associated with solar flares and in doing so have disregarded many events for which the whole range of frequencies does not show a large noise event. In those cases under consideration all frequencies show a response. I have tried to avoid the classification type II, III, IV, and so on, and have used instead slow-drift and fast-drift bursts. Also, I consider the starting time of the major noise increase at each frequency. In this way if the disturbance at any one frequency is caused by the particles, this should start when the initial edge of the particle cloud reaches the respective region of the corona. This is the only basis of my argument.

Pawsey: I am very much worried by an interpretation based on escape levels in a uniform corona, which is the model that radio astronomers have tried unsuccessfully to reconcile with observations for years. The results reported at this Symposium show conclusively that coronal irregularities must be a major feature. The report of ionospheric effects following certain bursts (presumed type III) is of major interest, and I hope that, when he has the necessary observations, Dr. Hartz will publish a careful statistical examination that will exclude the possibility of false identifications.

Hartz: I agree that structure in the corona could well affect my argument, but I do not know how large this effect might be. It seems unreasonable to assume that the accepted average electron distribution does not set the limiting " escape level" for the noise radiation. There is no real evidence to give the electron densities at these distances from the sun, other than an average density. The results on the ionospheric effects following the fast-drift bursts will be published soon. The effect itself is described in a paper by Collins and Reid (J. Atmos. Terr. Phys., in press). A paper on the association of the absorption effect with the solar bursts is being prepared for early publication.

Gold: I interpreted type II and III bursts as emission by streams of particles, each in the appropriate velocity range, after the flare of 1956 February 23 reported in The Observatory. I pointed out that such special conditions were imposed by the requirements of escape of the cosmic-ray particles from the solar fields, that we may well have cosmic-ray energies produced much more commonly but without the particles being able to reach the particular target of the earth. It appears that outbursts of sub-cosmic-ray-energy particles also occur for an event consisting of a large flare followed by a high-latitude ionospheric absorption of the type of the 1956 flare, but not accompanied by cosmic rays, as was observed last July. 
Roberts: I would like to point out that emission from the escape levels is not consistent with the observed spectra of type II bursts. On the plasma theory, with radiation coming from the escape level, the harmonic ratio should be very much less than 2 for bursts near the limb. This is not observed.

Hartz: Dr. Roberts' statement is based on an assumed generation process (plasma oscillation) and, in consequence, the electron density must be juggled in order to make the regions where the noise originates agree with coronal structures. In my argument I assume electron densities which are usually accepted, but take the region of origin to be at the escape envelope. By doing this I have been able to get good agreement in most cases between the deduced velocities for the particles and the sun-to-earth delay time observed between large solar events and subsequent terrestrial disturbances. So far, Wild has not been able to get any kind of agreement in specific cases.

Leona Marshall: All radiation, whether produced by synchrotron or plasma or Čerenkov mechanisms, is subject to requirements of total reflection resulting from decrease of plasma density with height in the corona. For the radiation that emerges at such an angle to the sun's radius vector that it can escape, we can discuss the intensity ratio of second to first harmonic. If the radiation is produced by synchrotron mechanism this ratio depends on energy of electrons producing the radiation and also on the angle between momentum vector of radiation and magnetic field direction. The observed ratio can be accounted for by many proper choices of these variables. On this account Dr. Roberts' objection is perhaps not necessarily valid.

Roberts: I believe a similar type of objection applies in the case of emission at harmonics of the gyro-frequency. 